\title{
Contraceptive use among women presenting to pharmacies for emergency contraception: an opportunity for intervention
}

\author{
Lucy Michie, ${ }^{1}$ Sharon T Cameron, ${ }^{2}$ Anna Glasier, ${ }^{3}$ Elizabeth Greed ${ }^{4}$
}

\begin{abstract}
${ }^{1}$ Clinical Research Fellow, Department of Reproductive and Developmental Sciences, University of Edinburgh, Edinburgh and Chalmers Sexual and Reproductive Health Centre, Edinburgh, UK

${ }^{2}$ Consultant Gynaecologist, Department of Reproductive and Developmental Sciences, University of Edinburgh, Edinburgh and Chalmers Sexual and Reproductive Health Centre, Edinburgh, UK

${ }^{3}$ Honorary Professor, Department of Reproductive and Developmental Sciences, University of Edinburgh, Edinburgh and London School of Hygiene and Tropical Medicine, London, UK

${ }^{4}$ Foundation Trainee Doctor, Great Western Hospital, Swindon, UK
\end{abstract}

\section{Correspondence to}

Dr Lucy Michie, Chalmers Sexual and Reproductive Health Centre, 2a Chalmers Street, Edinburgh EH3 9ES, UK; michieluc@yahoo.co.uk

Received 19 July 2013 Revised 26 November 2013 Accepted 3 December 2013 Published Online First 24 January 2014

CrossMark

To cite: Michie $L$

Cameron ST, Glasier A, et al.

$J$ Fam Plann Reprod Health

Care 2014:40:190-195.

\begin{abstract}
Objectives Most women who use emergency contraception (EC) do so because of unprotected sexual intercourse or condom failure and so remain at risk of pregnancy unless they commence an effective method of contraception. In Great Britain, increasingly women now choose to obtain EC from a pharmacy; however, pharmacists do not currently provide effective ongoing contraception. We sought to determine the views of women obtaining EC from pharmacies and clinicians working in sexual and reproductive health care $(\mathrm{SRH})$ about the possibility of pharmacists providing a temporary supply of a progestogen-only pill (POP) together with EC.
\end{abstract}

Methods Self-administered, anonymous questionnaires of (1) women requesting EC from pharmacies in Edinburgh, Scotland and (2) SRH clinicians attending a major UK scientific meeting.

Results A total of 211/232 women completed questionnaires in pharmacies (a $91 \%$ response rate). Of those women not using a hormonal method of contraception at the time of EC ( $n=166 ; 79 \%)$, almost half (44\%) wished to use an effective method. Most women (64\%) agreed that the option of a pharmacist being able to supply a POP would have been helpful. Among the SRH clinicians, 110 completed questionnaires out of 150 distributed (a $73 \%$ response rate).

The majority of respondents (92\%) were positive about a pharmacist supplying a POP at the time of EC.

Conclusions $A$ reasonable proportion of women requesting $\mathrm{EC}$ would like to start using an effective contraceptive method. Both the women and the SRH clinicians we surveyed are positive about the option of a short supply of a POP being provided by the pharmacy in the UK together with EC.

\section{Key message points}

- Only a minority of women presenting for emergency contraception (EC) are using an effective method of contraception at the time.

- Many women presenting for EC wish to use effective ongoing contraception and welcome the possibility of a supply of the progestogen-only pill (POP) from the pharmacist following EC.

- The majority of clinicians working in sexual and reproductive health care are positive about the possibility of pharmacists providing a limited supply of the POP at the time of EC.

\section{INTRODUCTION}

The majority of women who require emergency contraception (EC) do so following unprotected sexual intercourse (UPSI) or an accident when using a condom. ${ }^{1}{ }^{2}$ A smaller proportion of women may have had a mishap with a hormonal method of contraception (e.g. missed pills). ${ }^{1}{ }^{2}$ Increasingly, women in Great Britain prefer to attend a pharmacy for EC rather than a sexual and reproductive health (SRH) service or general practitioner (GP). ${ }^{3}$ Levonorgestrel EC has been available free of charge without a prescription from pharmacies since 2008 in Scotland ${ }^{4}$ and 2011 in Wales. ${ }^{5}$ In a recent trial comparing two oral emergency contraceptives fewer than 3\% of women became pregnant, and so the vast majority of women remain at risk of pregnancy after they have used EC. ${ }^{6}$ 
A few women get pregnant in the same menstrual cycle from sexual intercourse after taking EC. In a meta-analysis that included 11 trials of just under 5000 women who had sexual intercourse after using EC but before the return of menses (i.e. in the same cycle) the relative risk of pregnancy was 2.67 (2.113.39) when compared with women who did not have sex after using EC. ${ }^{7}$ Starting an effective ongoing method of contraception after EC use is clearly important if women are to avoid unintended pregnancy. Community pharmacists in the UK and most other industrialised countries are usually unable to provide any ongoing contraception except condoms, which are available for purchase. Two mystery shopper studies have shown that while pharmacists are good at adhering to protocols for providing EC, only a minority of them provide women with advice about ongoing contraception. ${ }^{8} 9$

Little is known about the views of women who present for EC on the use of regular, effective methods of contraception. In order to determine such views, and estimate the proportion of women using EC that may wish to start a method of effective contraception, we designed a questionnaire for women to complete when they attended a pharmacy for EC. We also sought to determine the views of women attending for EC, and clinicians working in sexual and reproductive health care (SRH), on the possibility of a pharmacist being able to provide women with a limited supply of progestogen-only oral contraceptives at the time of EC, allowing the women time to arrange an appointment to obtain a long-term contraception method.

\section{METHODS}

Two separate one-page, self-completed questionnaires were designed: (1) a questionnaire offered to women presenting to any of nine community pharmacies with a request for EC in January 2013 and (2) a questionnaire distributed to delegates (SRH clinicians) at the Faculty of Sexual and Reproductive Healthcare (FSRH) UK scientific meeting in May 2011. A short introductory paragraph explained the purpose and anonymous nature of both questionnaires. Selected community pharmacies in the City of Edinburgh and Lothian region (in Scotland) had participated in a clinical study aimed at increasing the uptake of regular contraception after EC in 2012. ${ }^{10}$ Nine pharmacists participating in that study, who dispensed EC to more than 10 women per month, agreed to participate in the questionnaire study. Pharmacists in Scotland can prescribe and dispense EC to women free of charge under a preapproved patient group direction (PGD), although they are required to complete additional training related to sexual health and contraception to enable them to do so. ${ }^{11}$ Women were offered the questionnaire by the pharmacist at the time of EC consultation, and once completed placed it in a sealed collection box before leaving the pharmacy. All questionnaires were numbered to allow us determine the response rate. At the FSRH scientific meeting questionnaires were distributed during a plenary session and collected from delegates at the end of the session. Both questionnaires, which included limited demographic data, required simple tick box responses, however additional space was provided for free text comments in response to certain questions. Delegates were asked to indicate how they felt about a limited supply of a progestogen-only oral contraceptive pill (POP) being offered to women presenting for EC. Responses included extremely positive, positive, neutral, negative and extremely negative. For the purposes of analysis, extremely positive and positive results were combined, as were negative responses.

\section{Statistics}

All the data were coded and entered onto two separate databases using Microsoft Excel ${ }^{\mathrm{TM}}$. Data analysis was performed using the IBM Statistical Package for Social Sciences (SPSS) software V.18 (SPSS Inc., Chicago, IL, USA). Demographic data were obtained including means and standard deviations (SDs) where appropriate. To allow statistical comparison between age groups in the questionnaire conducted in the pharmacies four age groups were defined: 14-19, 20$24,25-34$ and 35 years and over. Comparisons were made using Chi square $\left(\chi^{2}\right)$ test or Fisher's exact test where appropriate if counts within the individual cells of the contingency table fell below 5. Statistical significance was deemed to be $p<0.05$.

\section{Ethical approval}

The questionnaire for women attending pharmacies for EC was reviewed by the scientific officer of the local research ethics committee, who confirmed that ethical approval was not required as the questionnaire was an opinion survey seeking views of patients on a health care issue. Ethical approval was not required for the questionnaire distributed at the scientific meeting as the responses were anonymous.

\section{RESULTS}

\section{Pharmacy questionnaire}

A total of 211 completed questionnaires were obtained from 232 distributed to women attending pharmacies for EC (a 91\% response rate). The mean age of respondents was 23 (range 14-48; SD 5.6) years. For 59 women $(28 \%)$ this was the first time they had taken EC; $151(72 \%)$ had used it before and one person $(0.5 \%)$ did not answer the question. Significantly more women aged $14-19$ years were using EC for the first time compared to women aged 35 years and over (Table 1 ). The mean number of episodes of ever-use of EC was 2 (range 1-7) and for use in the past 12 months was 1 (range 1-4). 
Article

Table 1 Previous use of emergency contraception (EC) by women attending a pharmacy for EC provision

\begin{tabular}{|c|c|c|c|c|c|c|c|}
\hline \multirow[b]{2}{*}{ Age (years) } & \multirow[b]{2}{*}{$n(\%)$} & \multicolumn{3}{|c|}{ First ever use $[\boldsymbol{n}(\%)]$} & \multicolumn{3}{|c|}{ Use in past 12 months $[n(\%)]$} \\
\hline & & Yes & No & Missing data & Yes & No & Missing data \\
\hline$\overline{14-19}$ & $59(28)$ & $27(46)^{*}$ & $32(54)$ & 0 & $24(41)$ & $32(54)$ & $3(5)$ \\
\hline $20-24$ & $83(39)$ & $20(24)$ & $62(75)$ & 1 (1) & $23(28)$ & $50(60)$ & $10(12)$ \\
\hline $25-34$ & $58(27)$ & $11(19)$ & $47(81)$ & 0 & $20(34)$ & $33(57)$ & $5(9)$ \\
\hline$\geq 35$ & $11(5)$ & $1(9)^{*}$ & $10(91)$ & 0 & $3(27)$ & $8(73)$ & 0 \\
\hline Total & $211(99)$ & $60(28)$ & $151(72)$ & $1(0.5)$ & $70(33)$ & $123(58)$ & $18(9)$ \\
\hline
\end{tabular}

${ }^{*} p=0.006$.

The majority of women $(n=140 ; 66 \%)$ were using condoms as their contraceptive method at the time of requesting EC, whilst 45 (21\%) were using a hormonal method and 26 (12\%) were using no contraception (Figure 1). The majority of respondents $(n=163$; $77 \%$ ) stated they were currently in a sexual relationship at the time of using EC. Almost one-third of respondents $(n=66 ; 31 \%)$ required EC on this occasion because of UPSI, whilst almost half of them $(n=99 ; 47 \%)$ reported a condom failure (Table 2$)$.
There were no significant associations between respondents' age and method of contraception used, reason for use of EC or relationship status.

All the women were asked if they would like to start using a regular method of contraception other than condoms and, if so, from where they would choose to obtain it. Of the 166 women who were not already using a hormonal method of contraception at time of EC, $73(44 \%)$ women would like to do so (Figure 1). Most commonly women would choose to
Questionnaire respondents

Contraception at time of using EC

Would they like to start using a regular method of contraception?

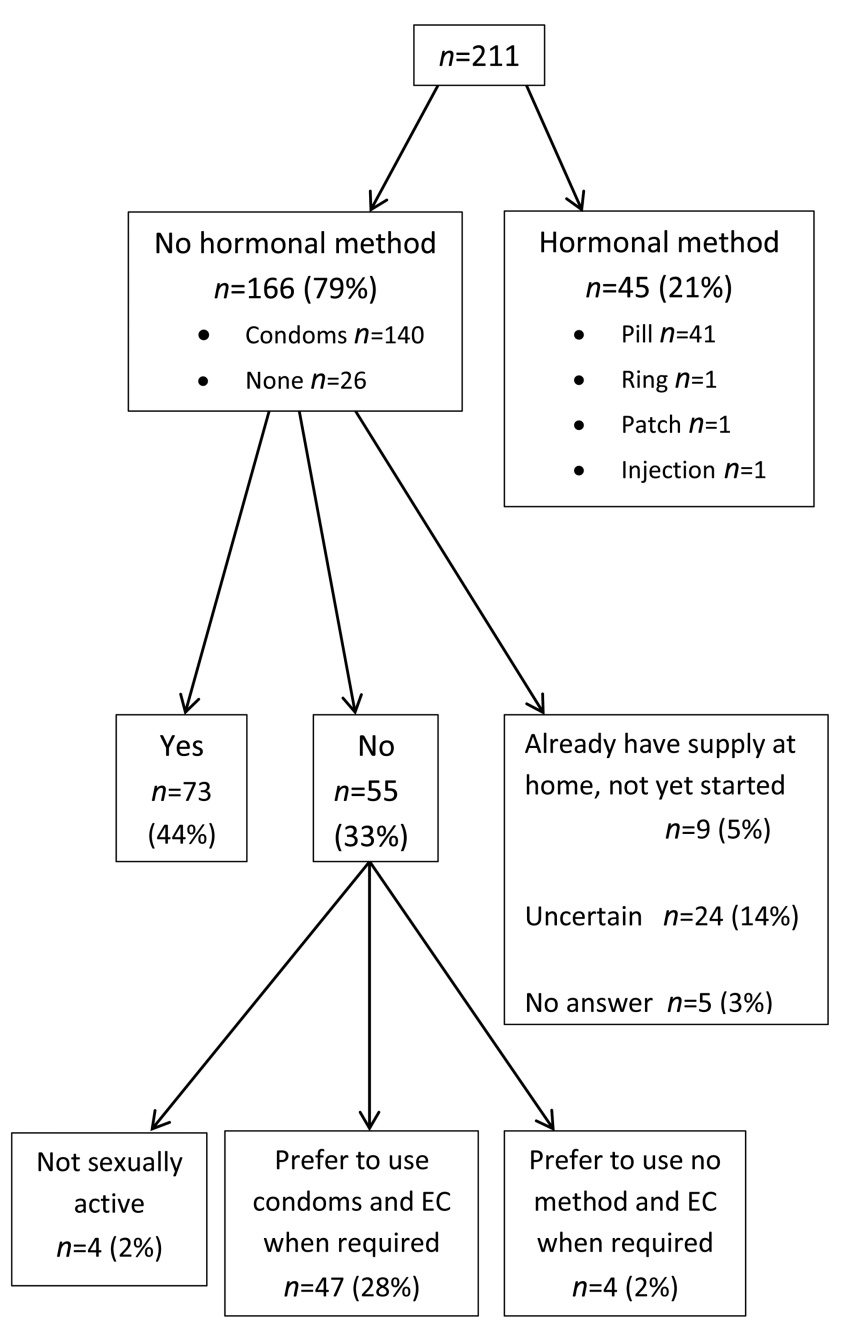

Figure 1 Flowchart of contraceptive use on presentation at a pharmacy for emergency contraception (EC) provision and interest in the use of a regular method of contraception. 
Table 2 Relationship status and reason for emergency contraception (EC) use by women attending a pharmacy for EC provision

\begin{tabular}{|c|c|c|c|c|c|c|c|c|}
\hline \multirow[b]{2}{*}{ Age (years) } & \multirow[b]{2}{*}{$n(\%)$} & \multicolumn{3}{|c|}{ In a sexual relationship [n(\%)] } & \multicolumn{4}{|c|}{ Reason for EC use $[n(\%)]$} \\
\hline & & Yes & No & Unsure & UPSI & Condom failure & Forgot regular method & Missing data \\
\hline$\overline{14-19}$ & $59(28)$ & $43(73)$ & $11(19)$ & $5(8)$ & $23(39)$ & $25(42)$ & $11(19)$ & 0 \\
\hline $20-24$ & $83(39)$ & $64(77)$ & $17(20)$ & $2(2)$ & $26(31)$ & $37(45)$ & $19(23)$ & $1(1)$ \\
\hline $25-34$ & $58(27)$ & $48(83)$ & $7(12)$ & $3(5)$ & $15(26)$ & $29(50)$ & $13(22)$ & $1(2)$ \\
\hline$\geq 35$ & $11(5)$ & $8(73)$ & $2(18)$ & $1(9)$ & $2(18)$ & $8(73)$ & $1(9)$ & 0 \\
\hline Total & $211(99)$ & $163(77)$ & $37(18)$ & $11(5)$ & $66(31)$ & $99(47)$ & $44(21)$ & $2(1)$ \\
\hline
\end{tabular}

UPSI, unprotected sexual intercourse.

obtain contraception from their GP (family doctor) ( $n=80 ; 48 \%)$, while $12(7 \%)$ would attend a family planning clinic; four (2\%) did not state a preference; seven $(4 \%)$ would attend a sexual health service for young people and seven (4\%) were unsure where they would go. One-third $(n=52 ; 31 \%)$ of the women chose not to answer this question. Three women stated they would choose to obtain contraception from a pharmacy, while one woman stated she wished to go "somewhere no one knows her" to obtain contraception.

Women were asked if it would have been helpful for the pharmacist to provide a 1-month supply of a POP to allow them time to attend elsewhere for ongoing contraception. The majority of respondents $(n=135 ; 64 \%)$ agreed that this would have been helpful, 25 (12\%) women felt it would not be, 38 $(18 \%)$ were unsure and $13(6 \%)$ did not respond. Significantly more young women (aged 14-19 years) felt a supply of a POP would have been helpful, compared to women aged 35 years and over $(80 \%$ vs $18 \% ; p=0.002)$. Fifteen women added free text comments to their response to this question. Five women made positive comments stating that this was a good idea, and three women commented that it would be helpful as it was difficult to obtain an appointment with their GP to discuss contraception. The reasons given by the seven women who stated that it may not be helpful included problems using the POP in the past $(n=2)$; a wish to avoid a hormonal method $(n=1)$; concern about possible side effects $(n=2)$; a preference to discuss contraceptive methods with a doctor $(n=1)$ and concern that a medical condition they had might contraindicate use of the POP $(n=1)$.

\section{Scientific meeting questionnaire}

A total of 110 questionnaires were completed from 150 distributed at the FSRH scientific meeting (a $73 \%$ response rate). The majority of respondents were female $(88 \%)$ and $90 \%$ were doctors (Table 3$)$. When asked how they felt about the concept of a pharmacist being able to provide a 28-day supply of a POP at the time of EC the majority $(n=101 ; 92 \%)$ felt positively about this, whilst six $(5 \%)$ were neutral and three (3\%) were negative. There were no statistically significant differences in views about this concept so far as gender, age group and work roles were concerned. Respondents were invited to provide additional free comments about this concept. Six made comments reaffirming their view that this is a good idea with benefits for women. Concerns expressed included that this may lead to a decrease in the use of long-acting reversible methods of contraception (LARCs) $(n=2)$ or an increase in the use of EC $(n=2)$, and two delegates stated that they would still prefer women to be reviewed by a medical professional in order to discuss all available methods of contraception and undergo testing for sexually transmitted infections where appropriate. Concern was also expressed that pharmacists would require adequate training to enable them to dispense the POP and advise women correctly $(n=4)$.

\section{DISCUSSION}

Our study confirmed previous findings that the majority of women presenting for EC do so either following UPSI or as a result of condom failure. ${ }^{12}$ Given the small number of women using effective contraception at the time of EC, and the fact that more than $75 \%$ of the survey respondents were in an ongoing sexual relationship, we can assume that the majority of them remained at risk of unintended pregnancy. Reassuringly, however, our study did identify that

Table 3 Demographics of respondents at a Faculty of Sexual and Reproductive Healthcare scientific meeting

\begin{tabular}{lr}
\hline Demographic & $\boldsymbol{n}(\%)$ \\
\hline Gender & \\
$\quad$ Female & $97(88)$ \\
Male & $13(12)$ \\
Age range (years) & \\
$<30$ & $3(3)$ \\
$31-40$ & $16(15)$ \\
$41-50$ & $49(45)$ \\
$>50$ & $42(38)$ \\
Occupation & \\
Doctor & $99(90)$ \\
Nurse & $10(9)$ \\
Other & $1(1)$ \\
\hline
\end{tabular}


almost half of the women not already doing so would wish to use an ongoing method of contraception, suggesting that there is potential to target this sizeable group of women to increase contraceptive use following EC. Our results suggest that significantly more young women $(<20$ years of age) were using EC for the first time compared to older women $(>35$ years of age), and that significantly more women welcomed the option of a supply of a POP at the time of EC. Although the number of women aged 35 years or over was small and there is the possibility this may have resulted by chance, it suggests that younger and potentially more vulnerable women may be receptive to simple interventions to increase contraceptive uptake.

Research has shown that pharmacists are good at supplying EC, and that women rate community pharmacy EC services highly. ${ }^{9}{ }^{12}$ However, research has also identified that pharmacists are not particularly good at providing advice about ongoing contraception, and some women have expressed concerns about receiving advice in the pharmacy about future contraception. 91213 A recent study in London, UK concluded that when pharmacists were trained to provide oral contraception by means of an approved PGD they were competent in doing so and women were satisfied with this additional service. ${ }^{14}$ Simple interventions within the pharmacy that may encourage and help women to start effective contraception after EC have also been the subject of recent research. ${ }^{15}$ We considered the possibility of a pharmacist providing a limited supply of a POP, allowing women time to arrange an appointment with a health care professional to discuss contraception further. We suggested offering a POP rather than a supply of the combined oral contraceptive pill (COC) since the list of contraindications to a POP is very small, making it more suitable for pharmacy provision. ${ }^{16}$ The concept was welcomed by the majority of women presenting for EC and also by the SRH clinicians. Women commented on the difficulty in obtaining an appointment with their GP, and that this could serve as interim measure in such situations.

Obviously not all women who use EC wish to commence a regular method of contraception. A proportion of women will inevitably make an informed choice to use condoms or no contraception, and interventions like this are unlikely to impact on this group of women. ${ }^{1} 1517$ As with other studies, some women had concerns about obtaining a limited supply of a POP from the pharmacist; ${ }^{12}$ however, providing reassurance to women may allay some of these concerns. Women can be reassured of the very small daily dose of progestogen in a POP compared to the dose in EC, and its safety compared with the COC. A small number of health professionals expressed concern that such interventions may decrease the use of the most effective LARC methods and thus preferred that women attended a clinic to discuss such methods.
However, we know that a large number of women now seek EC from community pharmacies ${ }^{3}$ and that they rate such services highly. ${ }^{12}$ Moreover, even when women attend specialist services for EC, almost threequarters of them leave without effective contraception, let alone a long-acting method. ${ }^{1}$ Therefore, we need to establish ways in which we can help women access ongoing contraception after obtaining EC from a pharmacy.

A limitation to our study is the lack of demographic data available from both our study populations. Short, anonymous questionnaires were used in order to encourage a high response rate and we chose to limit the amount of demographic data sought. However, the survey was conducted in several large pharmacies located within a large city, with a response rate close to $100 \%$, so we would hope that our study population is close to being representative of most women presenting for EC in Scotland.

Encouragingly, many women presenting for EC would wish to use effective ongoing contraception if they do not already do so, and would welcome a simple intervention in the pharmacy to help them do so. SRH clinicians, who are experts in contraception, are also positive about such an intervention. By helping women to obtain ongoing methods of effective contraception at time of using EC we may succeed in preventing more unintended pregnancies for more women.

Acknowledgements The authors are grateful to the organisers of the Faculty of Sexual and Reproductive Healthcare annual scientific meeting in Liverpool for allowing them to distribute the questionnaire, and to the pharmacists who agreed to give this questionnaire to women presenting for emergency contraception to their pharmacy: Shazad Aziz, Caroline Barnes, Jane Dewart, Michele Hamilton, Linzi Jack, Izabela Kalka, Peter Tinkler, Gill Toohie, Alison Wallace and Fiona Watson.

Competing interests None.

Provenance and peer review Not commissioned; externally peer reviewed.

\section{REFERENCES}

1 Cameron ST, Glasier A, Johnstone A, et al. Ongoing contraception after use of emergency contraception from a specialist contraceptive service. Contraception 2011;84:368-371.

2 Lo SST, Ho PC. Changes in contraceptive choice after emergency contraception. Int J Gynecol Obstet 2012;118:223-226.

3 Marston C, Meltzer H, Majeed A. Impact on contraceptive practise of making emergency hormonal contraception available over the counter in Great Britain: repeated cross sectional studies. BMJ 2005;331:271-276.

4 Primary Care Division. Additional pharmaceutical services, minor ailment service and public health service directions, service specifications and payment arrangements. Letter to pharmacists. Edinburgh, UK: Scottish Government, 2008.

5 All Wales Sexual Health Network. Emergency contraception free of charge in pharmacies across Wales. http://www.wales. 
nhs.uk/sites $3 /$ news.cfm ?orgid $=485 \&$ contentid $=18771$ [accessed 27 June 2013].

6 Glasier A, Cameron ST, Fine PM, et al. Ulipristal acetate versus levonorgestrel for emergency contraception: a randomized non-inferiority trial and meta-analysis. Lancet 2010;375:555-562.

7 Cheng L, Che Y, Gulmezoglu AM. Interventions for emergency contraception. Cochrane Database Syst Rev 2012;8:CD001324.

8 Anderson C, Bissell P. Using semi covert research to evaluate an emergency hormonal contraception service. Pharm World Sci 2004;26:102-106.

9 Glasier A, Manners R, Loudon JC, et al. Community pharmacists providing emergency contraception give little advice about future contraceptive use. A mystery shopper study. Contraception 2010;82:538-542.

10 Michie L, Cameron ST, Glasier A, et al. Initiatives to improve uptake of effective contraception after emergency contraception from the pharmacy: a pilot study. $\mathrm{Br} \mathrm{J}$ Obstet Gynaecol 2013;120:578. doi:10.1111/1471-0528.12354.

11 NHS Education for Scotland. Emergency hormonal contraception. E learning resource. http://www.nes.scot.nhs.uk/ education-and-training/by-discipline/pharmacy/ about-nes-pharmacy/educational-resources/resources-by-topic/ sexual-and-reproductive-health/reproductive-health/ contraception/emergency-hormonal-contraception.aspx [accessed 24 November 2013].

12 Anderson C, Blenkinsopp A. Community pharmacy supply of emergency hormonal contraception: a structured literature review of international evidence. Hum Reprod 2006;21:272-284

13 Black K, Mercer CH, Kubba A, et al. Provision of emergency contraception: a pilot study comparing access through pharmacies and clinical settings. Contraception 2008;77:181-185.

14 Parsons J, Adams C, Aziz N, et al. Evaluation of a community pharmacy delivered oral contraception service. J Fam Plann Reprod Health Care 2013;39:97-101.

15 Chin-Quee DS, Wedderburn M, Otterness C, et al. Bridging emergency contraceptive pill users to regular contraception: results from a randomized trial in Jamaica. Contraception 2010;81:133-189.

16 World Health Organization. Medical Eligibility Criteria for Contraceptive Use (4th edn). 2009. http://whqlibdoc.who.int/ publications/2010/9789241563888_eng.pdf [accessed 15 July 2013].

17 Chin-Quee DS, Hinson L, L'Engle KL, et al. Bridge over troubled waters: considerations in transitioning emergency contraceptive users to hormonal methods. Contraception 2012;85:363-368. 